\title{
Ground Glass Appearance on CT Thorax - Atypical Feature of Scrub Typhus - A Case Report
}

\author{
Kolluru Karthik Raja ${ }^{1}$, Sunil Kumar², A.H. Inamdar ${ }^{3}$, Amrutha Garikapati, Sameera Dhronamraju ${ }^{5}$ \\ 12,3, 4, 5 Department of General Medicine, Jawaharlal Nehru Medical College, Datta Meghe Institute of \\ Medical Sciences (Deemed to Be University), Wardha, Maharashtra, India.
}

\section{PRESENTATION OF CASE}

A 30-years-old male presented with 9 days history of high-grade fever with rash all over body, generalized body ache, altered sensorium since 4 days and with 3 days history of breathlessness. He had no history of headache, cough, vomiting, and diarrhea. He had no history of hypertension, diabetes, tuberculosis, asthma or thyroid disorders. On examination, he was ectomorphic, febrile with a pulse rate of $124 \mathrm{bpm}$, his BP was $130 / 80 \mathrm{~mm} \mathrm{Hg}$. He had petechial rash all over the body and icterus was present. No lymph nodes were palpable. On abdominal examination, his liver and spleen were palpable. There was no tenderness / guarding / rigidity over abdomen.

His Laboratory values were - Hemoglobin $14.7 \mathrm{mg} / \mathrm{dL}$, haematocrit $43 \mathrm{mg} / \mathrm{dL}$, platelets count was $27,000 / \mathrm{mm}$, white blood cell (WBC) was 14,300 cells / cumm with $84 \%$ neutrophils, $2 \%$ eosinophil, $4 \%$ monocyte. Biochemical lab values were serum albumin $3.0 \mathrm{gm} / \mathrm{dL}$, serum proteins $6.4 \mathrm{gm} / \mathrm{dL}$, aspartate aminotransferase and aminotransferase were 160 and $153 \mathrm{IU} / \mathrm{L}$, blood urea $229 \mathrm{mg} / \mathrm{dL}$, creatinine $3.5 \mathrm{mg} / \mathrm{dL}$, serum $\mathrm{Na}^{+} \& \mathrm{~K}^{+}$were 154 and $4.1 \mathrm{meq} / \mathrm{L}$. cerebrospinal fluid analysis reveals white blood cells $=1-2$ cells $/$ hpf, total leucocyte count $=$ Approximately 13 cells $/$ cumm, differential leucocyte count $=$ Polymorphs $=85 \%$, Lymphocytes $=15$ $\%$, cerebrospinal fluid glucose $75 \mathrm{mg} / \mathrm{dL}$, lactate dehydrogenase 85, cerebrospinal fluid protein $162 \mathrm{mg} / \mathrm{dL}$, PH 7.5. Urine analysis reveals mild proteinuria. Peripheral smear shown normocytic, mildly hypochromic red blood cells with platelets reduced in smear with neutrophilic leukocytosis with mild shift to left up to band forms. Blood and urine culture suggestive of no microbial growth. COVID-19 test by reverse transcriptase polymerase chain reaction (RT-PCR) was negative. Scrub typhus by Rapid detection kit test (a solid phase immune chromatographic assay) was strongly positive. Dengue serology and leptospira was negative. Ultrasound abdomen and pelvis revealed hepatomegaly with coarse echo texture with mild splenomegaly with bilaterally raised cortical echo texture. Chest x-ray revealed bilateral ground glass opacities in lower lung fields. Magnetic resonance imaging brain imaging revealed normal study. High-resolution computerized tomography thorax was suggestive of consolidation with surrounding ground glass opacities in bilateral posterior basal segments (Figure 1).

Scrub typhus fever is commonly undiagnosed due to its unusual presentation. Ground glass opacities in computed tomography thorax is one of the rare findings in a patient with scrub typhus infection. We reported a young male patient, admitted with chief complaints of fever with rash and breathlessness. Patient was positive for scrub typhus infection. Later he underwent CT thorax which was suggestive of consolidation with surrounding ground glass opacities in bilateral posterior basal segments.

Scrub typhus infection is a zoonotic disease caused by rickettsiae Orientia tsutsugamushi which is mainly confined to tropical region. ${ }^{1}$ Diagnosis is commonly delayed due to unusual presentation, restricted mindfulness, and lack of diagnostic facilities. ${ }^{2}$ Clinical features include fever, headache, generalized body ache, lymphadenopathy, cough, vomiting, diarrhea and rash. If patient was untreated or delayed diagnosis leads to complications like acute respiratory distress syndrome (ARDS), acute renal failure, meningoencephalitis, gastrointestinal bleeding (GI) bleeding and coagulopathy. ${ }^{3}$

Scrub typhus infection is an easily treatable condition, but sometimes it may lead to serious complications, if there is delay in diagnosis. We reported a patient who is positive for scrub typhus infection presented with ground glass appearance on CT thorax.

\author{
Corresponding Author: \\ Dr. Sunil Kumar, \\ Professor, \\ Department of Medicine, \\ Jawaharlal Nehru Medical College, \\ Datta Meghe Institute of Medical Sciences \\ (Deemed to Be University), Wardha, \\ Maharashtra, India. \\ E-mail: sunilkumarmed@gmail.com
}

DOI: $10.14260 / j e m d s / 2020 / 877$

How to Cite This Article:

Raja KK, Kumar S, Inamdar AH, et al. Ground glass appearance on CT thorax atypical feature of scrub typhus - a case report. J Evolution Med Dent Sci 2020;9(52):4012-4013, 10.14260/jemds/2020/877

Submission 04-08-2020,

Peer Review 02-11-2020,

Acceptance 09-11-2020,

Published 28-12-2020.

Copyright (c) 2020 Kolluru Karthik Raja et al. This is an open access article distributed under Creative Commons Attribution License [Attribution 4.0 International (CC BY 4.0)] 


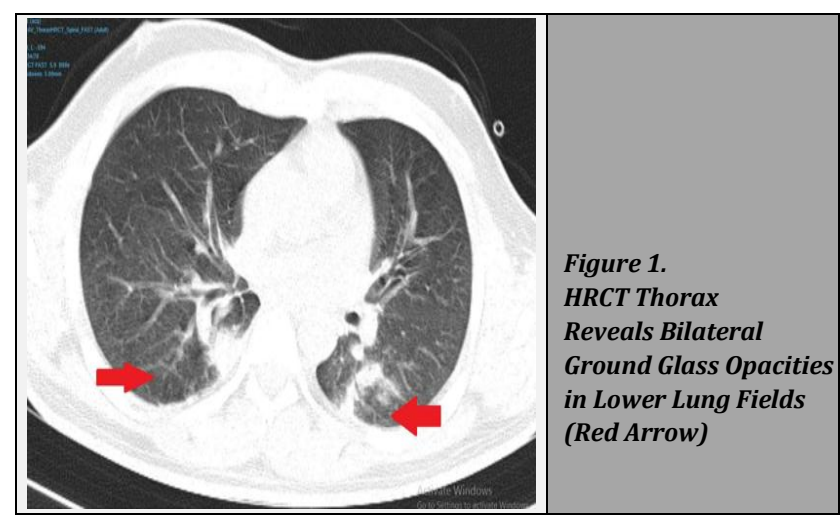

DISCUSSION OF MANAGEMENT

Patient was treated with injectable doxycycline, piperacillin, tazobactam, levofloxacin, intravenous fluids and other supportive management. Patient's general condition improved both clinically and radiologically and got discharged.

\section{DISCUSSION}

Scrub typhus infection caused by a mite Orientia tsutsugamushi is mostly confined to tropical region. It is estimated that one million people were prone to scrub typhus infection annually throughout globe. ${ }^{4}$ Eschar is the most distinctive finding in scrub typhus. It is a dark coloured, necrotic lesion which resembles a cigar burn usually found in skin which is thin, wrinkled or moist. ${ }^{5}$ Sites involved in the development of eschar are axilla, groin and breast. Generally eschar is demonstrable in half of patients who were certain for scrub typhus infection.

Scrub typhus infection presents as non-specific febrile illness associated with headache, generalized body ache, lymphadenopathy, cough, vomiting, diarrhea and rash. If untreated or diagnosis is delayed, patient may land in multiple organ dysfunction syndrome (MODS). Pathologic findings of scrub typhus have been described by vasculitis of the microvasculature in the involved organ resulting from a direct invasion of the mite (Orientia tsutsugamushi).

Lungs are commonly involved in patients of scrub typhus presenting with MODS. The chief respiratory complaints include cough and dyspnoea. Interstitial pneumonia in patients with scrub typhus infection commonly related with severity of disease. ARDS is rare and a serious complication of scrub typhus. The most commonly noted radiological findings of lung parenchyma includes interstitial pneumonia, reticulonodular opacities, consolidation, septal lines and hilar lymph nodes enlargement.6,7 Griffth et al. in a case study reported that patients of scrub typhus infection, respiratory organ dysfunction are predominantly noted.

Diffuse ground glass opacity is a non-specific finding in chest $\mathrm{x}$ rays and CT Thorax consists of hazy opacity without obscuring the underlying bronchial structures or pulmonary vessels indicating partial filling in the lungs by exudates or transudates, as well as interstitial thickening or partial collapse of lung and is most commonly associated with wide spread inflammatory and infiltrative lung disorders like severe acute respiratory distress (SARS) tuberculosis, pneumocystis jirovecii, cytomegalovirus, chronic interstitial lung diseases, and acute alveolar diseases. Focal Ground glass opacities (non-solid / part-solid nodules) are demarcated areas of hazy lung opacities which are associated with early stages of bronchioloalveolar carcinoma.

Gold standard serological marker for diagnosing scrub typhus infection is immunofluorescence assay. However, its use is limited due to cost and technical expertise. IgM (ELISA) enzyme-linked immunoassay, is the routinely used test for screening and confirming the diagnosis. Treatment for scrub typhus includes doxycycline, chloramphenicol, and azithromycin.

\section{CONCLUSIONS}

In the current pandemic, any patient presenting with fever and ground glass opacities in CT thorax, apart from thinking of COVID-19, one should keep scrub typhus infection as differential diagnosis.

Financial or other competing interests: None.

Disclosure forms provided by the authors are available with the full text of this article at jemds.com.

Informed consent was obtained from the relatives.

\section{REFERENCES}

[1] Tilak R, Kunte R. Scrub typhus strikes back: are we ready? Med J Armed Forces India 2019;75(1):8-17.

[2] Vivekanandan M, Mani A, Priya YS, et al. Outbreak of scrub typhus in Pondicherry. J Assoc Physicians India 2010;58:24-8.

[3] Jeong YJ, Kim S, Wook YD, et al. Scrub typhus: clinical, pathologic and imaging findings. Radiographics 2007;27(1):161-72.

[4] Kelly DJ, Fuerst PA, Ching WM, et al. Scrub typhus: the geographic distribution of phenotypic and genotypic variants of orientia tsutsugamushi. Clin Infect Dis 2009;48(Suppl 3):S203-30.

[5] Hulkoti V, Dubey A, Kumar S, et al. Psychotic scrub typhus: a case report. Medical Science 2020;24(102):472-74.

[6] Choi YH, Kim SJ, Lee JY, et al. Scrub typhus: radiological and clinical findings. Clin Radiol 2000;55(2):140-4.

[7] Kashyap S, Gautam N, Kanga A, et al. Pulmonary manifestations in patients with scrub typhus presenting to a tertiary care hospital of Northwest Himalayan region of India. Chest Infections 2012;142(4):218A. 